\title{
Optical Decentration
}

National Cancer Institute

\section{Source}

National Cancer Institute. Optical Decentration. NCI Thesaurus. Code C62891.

Problem associated with being off-center of optical lenses. 